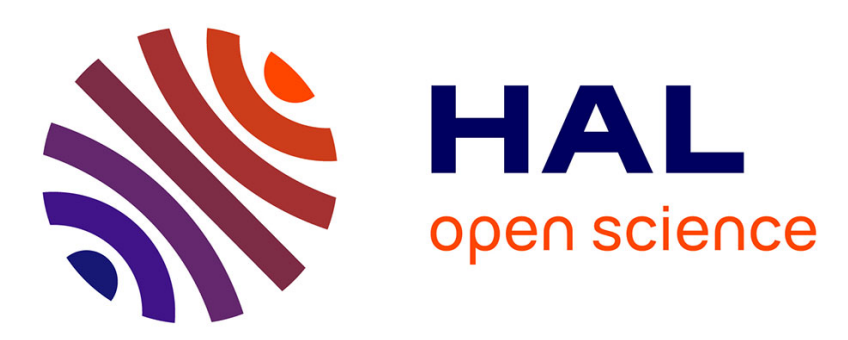

\title{
Calculation of optical phase jitter in dispersion-managed systems by use of the moment method
}

\author{
M. Hanna, D. Boivin, P.A. Lacourt, Jean-Pierre Goedgebuer
}

\section{To cite this version:}

M. Hanna, D. Boivin, P.A. Lacourt, Jean-Pierre Goedgebuer. Calculation of optical phase jitter in dispersion-managed systems by use of the moment method. Journal of the Optical Society of America B, 2004, 21 (1), pp.24-28. 10.1364/JOSAB.21.000024 . hal-00096895

\section{HAL Id: hal-00096895 \\ https://hal.science/hal-00096895}

Submitted on 12 May 2021

HAL is a multi-disciplinary open access archive for the deposit and dissemination of scientific research documents, whether they are published or not. The documents may come from teaching and research institutions in France or abroad, or from public or private research centers.
L'archive ouverte pluridisciplinaire HAL, est destinée au dépôt et à la diffusion de documents scientifiques de niveau recherche, publiés ou non, émanant des établissements d'enseignement et de recherche français ou étrangers, des laboratoires publics ou privés.

\section{(c)(1)}

Distributed under a Creative Commons Attribution| 4.0 International License 


\title{
Calculation of optical phase jitter in dispersion- managed systems by use of the moment method
}

\author{
Marc Hanna, David Boivin, and Pierre-Ambroise Lacourt \\ GTL-CNRS Telecom, Unité Mixte de Recherche du Centre National de la Recherche Scientifique 6603, 2-3 rue \\ Marconi, 57070 Metz, France \\ Jean-Pierre Goedgebuer \\ Laboratoire d'optique P. M. Duffieux, Unité Mixte de Recherche, Centre National de la Recherche Scientifique 6603, \\ Route de Gray 25030 Besançon Cedex, France
}

\begin{abstract}
Optical phase jitter limits the performance of amplified differential-phase-shift-keyed optical communication systems. We propose an approach to evaluate the phase jitter for arbitrary pulses in dispersion-managed links based on the moment method. This calculation requires only the knowledge of the unperturbed optical signal, therefore avoiding computationally intensive Monte Carlo simulations. We apply this method to a dispersion-managed soliton system and a quasi-linear dispersion-compensated channel and demonstrate its validity by comparing the obtained results with Monte Carlo simulations.
\end{abstract}

\section{INTRODUCTION}

There has recently been a renewed effort to develop coherent optical communication systems, particularly differential phase-shift keying ${ }^{1-3}$ (DPSK), which does not require a local oscillator to perform decoding. The motivation to use differential phase-shift keying in wavelengthdivision-multiplexed (WDM) systems is twofold. First, modulation formats based on phase show an increased robustness to nonlinear impairments such as cross-phase modulation ${ }^{4}$ and nonlinear polarization rotation, ${ }^{5}$ mostly because the time dependence of the optical power is deterministic and periodic. Second, coherent formats may allow a higher spectral efficiency, ${ }^{6}$ since both in-phase and quadrature dimensions of the signal space are available to encode information. Optical phase is also used in intensity-modulated direct-detection systems as an extra degree of freedom, for example, to provide better resistance to intrachannel four-wave mixing ${ }^{7}$ or to increase spectral efficiency in duobinary modulation.

The physical mechanism that limits performance in coherent systems is amplified-spontaneous-emission (ASE) noise-induced phase jitter. ${ }^{8}$ Analytic derivations of the phase uncertainty for soliton systems were carried out by use of perturbation theory and the variational method. These studies assume an analytically determined pulse shape and a constant-dispersion optical link. ${ }^{9,10}$ A semianalytic model of the phase jitter was recently proposed for dispersion-managed (DM) soliton transmission, ${ }^{11}$ based on a Gaussian ansatz for the optical field. Here we propose a semianalytic approach that is valid for arbitrary pulses in DM optical links, following the methodology presented in Ref. 12. The principle of this derivation is to split the propagation problem into its deterministic and random parts. The probabilistic aspect of the problem is solved analytically, thereby avoiding the computation of average quantities over a statistically significant number of noise sample functions. The deterministic part of the problem is carried out numerically by use of the split-step Fourier algorithm. The obtained unperturbed optical field is used together with the moment method $^{13}$ and statistical properties of the noise to evaluate phase jitter to first order. This approach is validated by comparison with direct Monte Carlo simulations in a DM soliton system and a quasi-linear channel, and both show excellent agreement. The computation time necessary to evaluate the impact of phase jitter on a communication system is thereby reduced from hours to seconds on current desktop computers. This method also provides some physical insight about the mechanisms that create phase jitter in the context of arbitrary optical pulses.

\section{DERIVATION OF THE PHASE JITTER}

Optical pulse propagation in the picosecond regime is described by the scalar nonlinear Schrödinger equation

$$
i \frac{\partial u}{\partial z}-\frac{\beta_{2}(z)}{2} \frac{\partial^{2} u}{\partial t^{2}}+\gamma(z)|u|^{2} u=i g(z) u+\hat{F}(z, t)
$$

where $u(z, t)$ is the pulse envelope in a comoving frame, $g(z)$ is the local net gain, and $\beta_{2}(z)$ is the local groupvelocity dispersion. The coefficient $\gamma=n_{2} \omega_{0} / c A_{\text {eff }}$ is the local nonlinearity, where $n_{2}$ is the Kerr nonlinear index, $\omega_{0}$ is the signal's central angular frequency, $c$ is the speed 
of light, and $A_{\text {eff }}$ is the fiber's effective cross section. The noise source term is described by its autocorrelation function

$$
\left\langle\hat{F}(z, t) \hat{F}^{*}\left(z^{\prime}, t^{\prime}\right)\right\rangle=2 g_{0} \hbar \omega_{0} n_{\mathrm{sp}}(z) \delta\left(z-z^{\prime}\right) \delta\left(t-t^{\prime}\right),
$$

where $n_{\mathrm{sp}}$ is the spontaneous emission factor, $g_{0}$ is the amplification coefficient inside the amplifier, and $\hbar \omega_{0}$ is the photon energy at the signal frequency. We define the following quantities averaged over time:

$$
\begin{aligned}
E & =\int_{-\infty}^{+\infty}|u|^{2} \mathrm{~d} t, \\
P & =\frac{1}{E} \int_{-\infty}^{+\infty}|u|^{4} \mathrm{~d} t, \\
\Phi & =\frac{1}{E} \int_{-\infty}^{+\infty}|u|^{2} \arg (u) \mathrm{d} t, \\
\Omega^{2} & =-\frac{1}{2 E} \int_{-\infty}^{+\infty} \frac{\left(u u_{t}^{*}\right)^{2}+\left(u^{*} u_{t}\right)^{2}}{|u|^{2}} \mathrm{~d} t,
\end{aligned}
$$

where the subscript $t$ denotes the partial time derivative. The quantities $E, P, \Phi$, and $\Omega^{2}$, respectively, represent the energy, power, phase, and square of the angular frequency of the optical field at a given distance. Differentiating $\Phi$ and $P$ with respect to $z$ and using the propagation equation (1), we obtain the dynamic equations

$$
\begin{aligned}
\frac{\mathrm{d} \Phi}{\mathrm{d} z}= & \gamma P-\frac{\beta_{2}}{2} \Omega^{2}+\frac{i}{E} \int_{-\infty}^{+\infty}[\arg (u)-\Phi] \\
& \times\left(u \hat{F}^{*}-u^{*} \hat{F}\right) \mathrm{d} t-\frac{1}{2 E} \int_{-\infty}^{+\infty} u^{*} \hat{F}+u \hat{F}^{*} \mathrm{~d} t \\
\frac{\mathrm{d} P}{\mathrm{~d} z}= & 2 g P+\frac{\beta_{2}}{E} \int_{-\infty}^{+\infty}|u|^{4}[\arg (u)]_{t t} \mathrm{~d} t \\
& +\frac{i}{E} \int_{-\infty}^{+\infty}\left(2|u|^{2}-P\right)\left(u \hat{F}^{*}-u^{*} \hat{F}\right) \mathrm{d} t
\end{aligned}
$$

If we assume that the pulse exhibits mostly linear chirp, then $\arg (u)(z, t) \approx \phi_{0}(z)+\phi_{2}(z)\left(t-t_{0}\right)^{2}$, where $t_{0}$ is the temporal position of the pulse, and these dynamic equations can be rewritten as ${ }^{14}$

$$
\begin{aligned}
\frac{\mathrm{d} \Phi}{\mathrm{d} z}= & -\beta_{2} \phi_{2} \Phi+\gamma P+\frac{i}{E} \int_{-\infty}^{+\infty}[\arg (u)-\Phi] \\
& \times\left(u \hat{F}^{*}-u^{*} \hat{F}\right) \mathrm{d} t-\frac{1}{2 E} \int_{-\infty}^{+\infty} u^{*} \hat{F}+u \hat{F}^{*} \mathrm{~d} t \\
\frac{\mathrm{d} P}{\mathrm{~d} z}= & 2\left(g+\beta_{2} \phi_{2}\right) P+\frac{i}{E} \int_{-\infty}^{+\infty}\left(2|u|^{2}-P\right) \\
& \times\left(u \hat{F}^{*}-u^{*} \hat{F}\right) \mathrm{d} t
\end{aligned}
$$

These equations have simple physical interpretations. The mean phase accumulates proportionally to power through self-phase modulation (SPM). Phase variations also arise from chirp fluctuations through group-velocity dispersion. The last two terms in Eq. (9) represent the direct contribution of noise to phase evolution. The power varies as a function of gain and dispersion. Noise also contributes directly to power variations through the last term on the right-hand side of Eq. (10). Using the method of variation of parameters, we can implicitly integrate the dynamic equation that governs the evolution of power:

$$
\begin{aligned}
P= & \left(P_{0}+i \int_{0}^{z}\left\{\frac{1}{E A_{1}} \int_{-\infty}^{+\infty}\left[\left(2|u|^{2}-P\right)\right]\right.\right. \\
& \left.\left.\times\left(u \hat{F}^{*}-u^{*} \hat{F}\right) \mathrm{d} t\right\} \mathrm{~d} z_{1}\right) A_{1},
\end{aligned}
$$

where

$$
A_{1}(z)=\exp \left[\int_{0}^{z} 2\left(g+\beta_{2} \phi_{2}\right) \mathrm{d} z_{1}\right] .
$$

Similarly, an implicit solution for the mean phase equation is given by

$$
\Phi=\Phi_{1}+\Phi_{2}+\Phi_{3}
$$

where

$$
\begin{aligned}
& \Phi_{1}=A_{2} \int_{0}^{z} \frac{\gamma P}{A_{2}} \mathrm{~d} z_{1} \\
& \Phi_{2}=i A_{2} \int_{0}^{z}\left\{\frac{1}{E A_{2}} \int_{-\infty}^{+\infty}[\arg (u)-\Phi]\right. \\
&\left.\qquad\left(u \hat{F}^{*}-u^{*} \hat{F}\right) \mathrm{d} t\right\} \mathrm{d} z_{1} \\
& \Phi_{3}=-\frac{A_{2}}{2} \int_{0}^{z}\left(\frac{1}{E A_{2}} \int_{-\infty}^{+\infty} u^{*} \hat{F}+u \hat{F}^{*} \mathrm{~d} t\right) \mathrm{d} z_{1} \\
& A_{2}= \exp \left(-\int_{0}^{z} \beta_{2} \phi_{2} \mathrm{~d} z_{1}\right)
\end{aligned}
$$

Under these assumptions, the noise-free field can be used in the right-hand sides of Eqs. (11) and (13), since the perturbed field adds only second- and higher-order corrections to the phase. The phase variance can be calculated by use of Eqs. (2), (11), and (13):

$$
\begin{aligned}
{\sigma_{\Phi}}^{2} & =\left\langle\Phi^{2}\right\rangle-\langle\Phi\rangle^{2} \\
& =\left\langle\Phi_{1}{ }^{2}\right\rangle+\left\langle\Phi_{2}{ }^{2}\right\rangle+\left\langle\Phi_{3}{ }^{2}\right\rangle+2\left\langle\Phi_{1} \Phi_{2}\right\rangle,
\end{aligned}
$$

where, defining the scalar product

$$
\left(\delta q_{i}, \delta q_{j}\right)=\int_{-\infty}^{+\infty} \delta q_{i} \delta q_{j}{ }^{*}+\delta q_{i}{ }^{*} \delta q_{j} \mathrm{~d} t
$$

and the functions

$$
\begin{aligned}
& \delta q_{1}=2 i \sqrt{g n_{\mathrm{sp}} \hbar \omega_{0}} \frac{2|u|^{2}-P}{E A_{1}} u, \\
& \delta q_{2}=2 i \sqrt{g n_{\mathrm{sp}} \hbar \omega_{0}} \frac{\arg (u)-\Phi}{E A_{2}} u,
\end{aligned}
$$




$$
\delta q_{3}=2 \sqrt{g n_{\mathrm{sp}} \hbar \omega_{0}} \frac{u}{E A_{2}}
$$

we find

$$
\begin{aligned}
\left\langle\Phi_{1}{ }^{2}\right\rangle= & A_{2}{ }^{2} \int_{0}^{z} \frac{\gamma A_{1}}{A_{2}} \int_{0}^{z_{1}} \frac{\gamma A_{1}}{A_{2}} \\
& \times \int_{0}^{z_{2}}\left(\delta q_{1}, \delta q_{1}\right) \mathrm{d} z_{3} \mathrm{~d} z_{2} \mathrm{~d} z_{1}, \\
\left\langle\Phi_{2}{ }^{2}\right\rangle= & \frac{A_{2}^{2}}{2} \int_{0}^{z}\left(\delta q_{2}, \delta q_{2}\right) \mathrm{d} z_{1}, \\
\left\langle\Phi_{3}{ }^{2}\right\rangle= & \frac{A_{2}{ }^{2}}{8} \int_{0}^{z}\left(\delta q_{3}, \delta q_{3}\right) \mathrm{d} z_{1}, \\
2\left\langle\Phi_{1} \Phi_{2}\right\rangle= & A_{2}{ }^{2} \int_{0}^{z} \gamma A_{1} \int_{0}^{z_{1}}\left(\delta q_{1}, \delta q_{2}\right) \mathrm{d} z_{2} \mathrm{~d} z_{1} .
\end{aligned}
$$

The cross products $\left\langle\Phi_{1} \Phi_{3}\right\rangle$ and $\left\langle\Phi_{2} \Phi_{3}\right\rangle$ are reduced to zero owing to orthogonality properties of the noise components. Equations (18)-(26) represent the main result of this paper and are valid for arbitrary pulse shapes and communication systems, provided that the chirp remains essentially linear throughout the propagation. Once the deterministic optical field is known, one can use it in the right-hand sides of Eqs. (23)-(26) to evaluate the phase jitter. The quantity $\left\langle\Phi_{1}{ }^{2}\right\rangle$ can be identified as the phase jitter induced by power fluctuations through SPM, $\left\langle\Phi_{2}{ }^{2}\right\rangle$ and $\left\langle\Phi_{3}{ }^{2}\right\rangle$ are direct contributions from the noise to phase jitter, and $\left\langle\Phi_{1} \Phi_{2}\right\rangle$ is the interference term between these two effects.

Note that our method takes into account only the interaction of a single pulse with ASE noise, neglecting other contributions to phase jitter that might arise in WDM systems, such as pulse-to-pulse intra- and interchannel interactions. This point will be further discussed at the end of Section 3.

\section{APPLICATION TO DISPERSION- MANAGED SYSTEMS}

We tested the validity of the moment-method approach by comparing it with direct Monte Carlo simulations. A single pulse was propagated by use of the split-step Fourier algorithm for two systems. The time, frequency, and distance resolutions were $500 \mathrm{fs}, 2 \mathrm{GHz}$, and $1 \mathrm{~km}$, respectively.

First, we considered a DM soliton link consisting of alternating spans of anomalous dispersion fiber with $D_{1}$ $=11(\mathrm{ps} / \mathrm{nm}) / \mathrm{km}$, length $L_{1}=30 \mathrm{~km}, \quad$ and $A_{\text {eff }}$ $=50 \mu \mathrm{m}^{2}$ and normal dispersion fiber with $D_{2}$ $=-10(\mathrm{ps} / \mathrm{nm}) / \mathrm{km}, L_{2}=30 \mathrm{~km}$, and $A_{\text {eff }}=50 \mu \mathrm{m}^{2}$. An unchirped sech-shaped pulse was launched at the midpoint of the normal dispersion span, with duration $\tau_{\mathrm{FWHM}}=20 \mathrm{ps}$ and peak power $P_{0}=7.2 \mathrm{~mW}$.

The second transmission system was a quasi-linear dispersion-compensated link formed by our alternating spans of standard single-mode fiber with $D_{1}=16(\mathrm{ps} / \mathrm{nm}) / \mathrm{km}, \quad L_{1}=50 \mathrm{~km}, \quad$ and $A_{\text {eff }}=80 \mu \mathrm{m}^{2}$ and dispersion-compensating fiber with $D_{2}$ $=-80(\mathrm{ps} / \mathrm{nm}) / \mathrm{km}, \quad L_{2}=10 \mathrm{~km}, \quad$ and $A_{\text {eff }}=45 \mu \mathrm{m}^{2}$, yielding a zero average dispersion. An unchirped Gaussian-shaped pulse was launched at the midpoint of the normal dispersion span, with duration $\tau_{\mathrm{FWHM}}$ $=20 \mathrm{ps}$ and peak power $P_{0}=0.1 \mathrm{~mW}$.

For both systems, lumped optical amplifiers were placed every $60 \mathrm{~km}$ along the link, with $n_{\mathrm{sp}}=1.5$. The average phase of the field was numerically evaluated by use of Eq. (5) over an ensemble of 500 simulations with different ASE noise to infer the phase variance. As only single pulses at a single wavelength were considered, patterning effects that may arise in real WDM communication systems are not studied here.

The phase standard deviation is plotted in Fig. 1 for propagation distances less than $1 \mathrm{Mm}$. For the quasilinear system, we observe a global linear dependance of the phase variance on propagation distance because terms $\left\langle\Phi_{2}{ }^{2}\right\rangle$ and $\left\langle\Phi_{3}{ }^{2}\right\rangle$ dominate owing to the low peak power. The phase uncertainty exhibits rapid oscillations that are imparted by the dynamics of the pulse inside a DM cell. As the variation of group-velocity dispersion is large in the case of the quasi-linear system, these oscillations are particularly strong. Our method reproduces these oscillation locations and amplitudes accurately. For the DM soliton system, the cubic dependence of the phase variance obtained in the case of constantdispersion soliton channels ${ }^{9}$ is still observed. This is due to the predominance of the $\left\langle\Phi_{1}{ }^{2}\right\rangle$ term, since the large optical peak power gives rise to significant SPM, thus transferring amplitude noise to the phase. Variations at the scale of the DM cell are small because of the low value of the dispersion map strength considered.

For distances over $1 \mathrm{Mm}$, we plotted only the phase standard deviation at the locations of optical amplifiers for clarity (Fig. 2). For the quasi-linear case, the moment-method results are in good agreement with Monte Carlo simulations for all distances. For the DM soliton case, we note that the oscillations of the phase uncertainty on a long-distance scale are reproduced with good accuracy. The moment method slightly overestimates the locations of these oscillations, probably because of second-order effects. We note that the phase jitter curves for the two systems cross at approximately $1 \mathrm{Mm}$. This observation can be physically interpreted as follows. Equations (20)-(22) show that the impact of noise on phase jitter is inversely proportional to the energy per

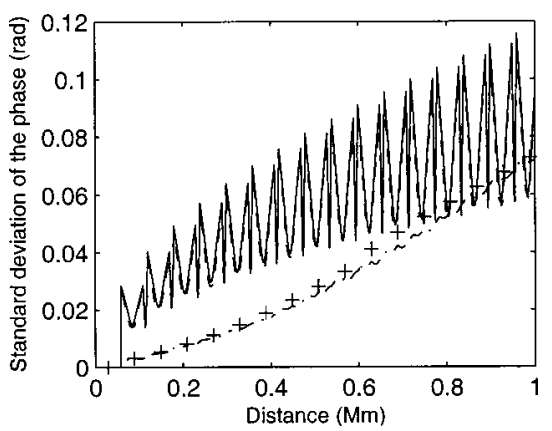

Fig. 1. Standard deviation of the phase as a function of distance over $1 \mathrm{Mm}$. Quasi-linear system: Monte Carlo (solid curve) and moment method (dashed curve). DM soliton system: Monte Carlo (dashed-dotted curve) and moment method (crosses). 


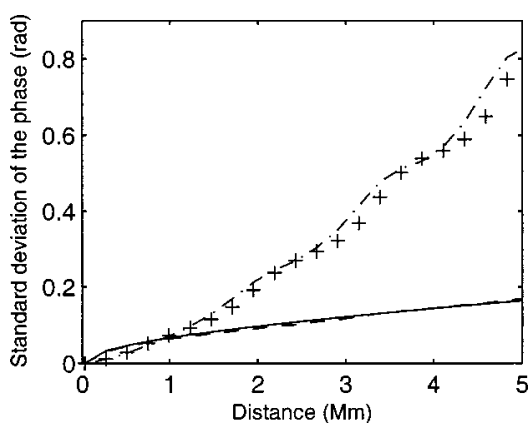

Fig. 2. Standard deviation of the phase as a function of distance over $5 \mathrm{Mm}$. Quasi-linear system: Monte Carlo (solid curve) and moment method (dashed curve). DM soliton system: Monte Carlo (dashed-dotted curve) and moment method (crosses).

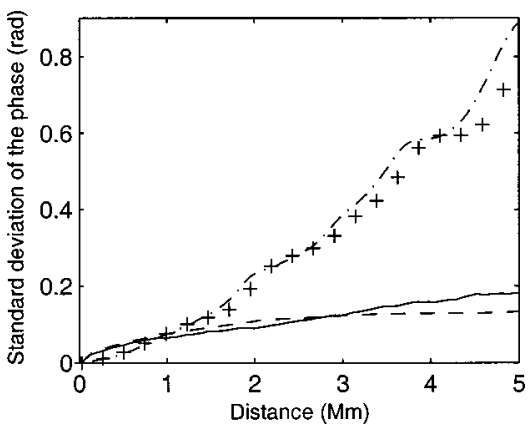

Fig. 3. Standard deviation of the phase as a function of distance over $5 \mathrm{Mm}$ for the multipulse simulation. Quasi-linear system: Monte Carlo (solid curve) and moment method (dashed curve). DM soliton system: Monte Carlo (dashed-dotted curve) and moment method (crosses).

pulse. In the short-distance case, the direct contribution from ASE to phase noise predominates. The quasi-linear system, operating at low energy per pulse, is more affected by noise. As the propagation distance grows, the contribution from nonlinear phase noise grows rapidly when the operating power is high. Therefore the phase jitter in the DM soliton case grows faster than in the quasi-linear case, resulting in a higher phase jitter for distances greater than $1 \mathrm{Mm}$. This shows the need for phase-control techniques, particularly in the case of highly nonlinear transmission systems.

We now discuss potential additional effects that might participate in the generation of phase jitter in WDM systems. Both four-wave mixing and cross-phase modulation result in interactions between pulses within a channel and from different channels. In DM systems, interchannel four-wave mixing is not phase matched, so that its overall influence on the propagation is small. The contribution from interchannel cross-phase modulation to phase jitter was shown to grow as the distance square in a constant-dispersion soliton system. ${ }^{15}$ However, it has a small impact on the performance of such systems because the random phase shifts experienced by successive pulses are highly correlated, so that they cancel when a differential modulation format is used.

The impact of intrachannel effects on phase jitter is not yet clearly assessed. To make a first evaluation of their eventual contribution, we made simulations of the propa- gation of seven pulses, each carrying a random phase chosen in the set $[0, \pi]$, in both of the aforementioned systems. The phase jitter for the central pulse was evaluated by use of Monte Carlo simulations, which take pulse-to-pulse interactions into account, and we also used the moment-method approach, which takes into account only the interaction with ASE noise. The isolation of the central pulse was performed by evaluation of all time integrals over the central bit slot only. The pulse repetition period was $T=100 \mathrm{ps}$, which yielded a moderate overlap in the DM soliton system (maximum FWHM pulse width-to-time slot ratio $\tau_{\max } / T=0.34$ ) and a strong overlap for the quasi-linear system $\left(\tau_{\max } / T=0.70\right)$.

The phase standard deviation as a function of distance at chirp-free points obtained in the multipulse simulation is plotted in Fig. 3. For the DM soliton system, the result is very similar to the single-pulse case, which indicates that intrachannel pulse-to-pulse interactions play a minor role in the generation of phase jitter for this link. For the quasi-linear case, the overlap is strong, so that the computation of the average phase of a pulse given by Eq. (5) is meaningful only at chirp-free points, when pulses are confined to their time slot. At other points, the contribution of neighboring pulses to the integral makes impossible the numerical evaluation of the average phase for a given pulse. This comment applies to both the Monte Carlo and the moment method. We observe that the Monte Carlo curve does not deviate significantly from the single-pulse case, indicating that intrachannel effects bring only a weak contribution to phase jitter. However, the moment method is less accurate than in the single-pulse case because the scalar products of Eqs. (23)-(26) are nonzero only at amplifier locations, where the overlap is maximum. Despite this effect, the moment method performs reasonably well. Thus, for the systems considered here, ASE noise and SPM appear to be the dominant mechanisms at the origin of phase jitter. However, the effects of intrachannel interactions in the general context of phase-encoded signals remain to be studied in depth.

\section{CONCLUSION}

We have derived an approach to compute phase jitter for arbitrary pulse shapes in dispersion-managed links based on the moment method. This approach assumes only that the pulse essentially exhibits linear chirp. The moment method was applied to DM soliton and quasi-linear dispersion-compensated communication systems. Comparisons with Monte Carlo simulations show an excellent agreement, and the moment method requires only a fraction of the computational time. Further research will include the study of the stabilizing influence of phasecontrol techniques such as in-line filtering ${ }^{13}$ and active ${ }^{2}$ and passive ${ }^{1}$ nonlinearity compensation on such systems.

\section{REFERENCES}

1. X. Liu, X. Wei, R. E. Slusher, and C. J. McKinstrie, "Improving transmission performance in differential phase-shiftkeyed systems by use of lumped nonlinear phase-shift compensation," Opt. Lett. 27, 1616-1618 (2002). 
2. C. Xu and X. Liu, "Postnonlinearity compensation with data-driven phase modulators in phase-shift keying transmission," Opt. Lett. 27, 1619-1621 (2002).

3. A. H. Gnauck, G. Raybon, S. Chandrasekhar, J. Leuthold, C. Doerr, L. Stulz, A. Agarwal, S. Banerjee, D. Grosz, S. Hunsche, A. Kung, A. Marhelyuk, D. Maywar, M. Movassaghi, X. Liu, C. Xu, X. Wei, and D. M. Gill, “2.5 Tb/s (64 $\times 42.7 \mathrm{~Gb} / \mathrm{s})$ transmission over $40 \times 100 \mathrm{~km} \mathrm{NZDSF}$ using RZ-DPSK format and all-Raman-amplified spans," in Optical Fiber Communications Conference, Vol. 70 of 2002 OSA Technical Digest Series (Optical Society of America, Washington, D.C., 2002), postdeadline paper FC2.

4. J. Leibrich, C. Wree, and W. Rosenkranz, "CF-RZ-DPSK for suppression of XPM on dispersion-managed long-haul optical WDM transmission on standard single-mode fiber," IEEE Photon. Technol. Lett. 14, 155-157 (2002).

5. C. Xu, X. Liu, and X. Wei, "Ultra-long haul DWDM transmission with differential phase-shift keying dispersionmanaged soliton," in 28th European Conference on Optical Communications 2002 (Institute of Electrical and Electronics Engineers, New York, 2002), paper 1.1.5.

6. G. P. Agrawal, "Coherent lightwave systems," in Fiber-Optic Communication Systems, K. Chang, ed. (Wiley, New York, 2002), p. 478

7. M. Forzati, J. Martensson, A. Berntson, and A. Djupsjobacka, "Reduction of intrachannel four-wave mixing using the alternate-phase RZ modulation format," IEEE Photon. Technol. Lett. 14, 1285-1287 (2002).
8. H. Kim and A. Gnauck, "Experimental investigation of the performance limitation of DPSK systems due to nonlinear phase noise," IEEE Photon. Technol. Lett. 15, 320-322 (2003).

9. M. Hanna, H. Porte, W. T. Rhodes, and J.-P. Goedgebuer, "Soliton optical phase control by use of in-line filters," Opt. Lett. 24, 732-735 (1999)

10. C. J. McKinstrie and C. Xie, "Phase jitter in single-channel soliton systems with constant dispersion," IEEE J. Sel. Top. Quantum Electron. 8, 616-625 (2002).

11. C. J. McKinstrie, C. Xie, and T. I. Lakoba, "Efficient modeling of phase jitter in dispersion-managed soliton systems," Opt. Lett. 27, 1887-1889 (2002).

12. V. S. Grigoryan, C. R. Menyuk, and R. M. Mu, "Calculation of timing and amplitude jitter in dispersion-managed optical fiber communications using linearization," J. Lightwave Technol. 17, 1347-1356 (1999).

13. C. J. McKinstrie, "Effects of filtering on Gordon-Haus timing jitter in dispersion-managed systems," J. Opt. Soc. Am. B 19, 1275-1285 (2002).

14. S. K. Turitsyn, T. Schäfer, and V. Mezentsev, "Generalized root-mean-square momentum method to describe chirped return-to-zero signal propagation in dispersion-managed fiber links," IEEE Photon. Technol. Lett. 11, 203-205 (1999).

15. C. J. McKinstrie, C. Xie, and C. Xu, "Effects of cross-phase modulation on phase jitter in soliton systems with constant dispersion," Opt. Lett. 28, 604-606 (2003). 